\title{
PRÉ-CONCENTRAÇÃO DE CHUMBO E CÁDMIO EM UM SISTEMA DE MICRO EXTRAÇÃo LÍQUIDO- LÍQUIDO E DETERMINAÇÃO POR ESPECTROMETRIA DE ABSORÇÃO ATÔMICA COM CHAMA
}

\author{
Eduardo Carasek* \\ Departamento de Química, Universidade Federal de Santa Catarina, CP 476, 88040-900 Florianópolis - SC \\ Jussara Wick Tonjes e Mauro Scharf \\ Departamento de Química, Universidade Regional de Blumenau, CP 1507, 89010-971 Blumenau - SC
}

Recebido em 27/6/01; aceito em 18/1/02

\begin{abstract}
A LIQUID-LIQUID MICROEXTRACTION SYSTEM FOR Pb AND Cd ENRICHMENT AND DETERMINATION BY FLAME ATOMIC ABSORPTION SPECTROMETRY. A method for determination of lead and cadmium in aqueous samples using solvent microextraction and dithizone as complexing agent with FAAS was developed. Solvent microextraction parameters were optimized. The effect of foreign ions on the extraction yields was studied. The extraction was carried out until the aqueous to organic phase ratio achieved a 250 fold preconcentration of metals. For preconcentration times of 4 min the $3 \sigma$ detection limits, relative standard deviations $(\mathrm{n}=7)$ and linear calibration ranges were $1.6 \mu \mathrm{g} \mathrm{L}^{-1}, 5.8 \%$ and $10.0-80.0 \mu \mathrm{g} \mathrm{L}^{-1}$ for lead and $11.1 \mathrm{ng} \mathrm{L}^{-1}, 5.9 \%$ and $0.3-3.0 \mu \mathrm{g} \mathrm{L}^{-1}$ for cadmium, respectively. The solvent microextraction procedure presented here was applied to the determination of lead and cadmium in natural waters.
\end{abstract}

Keywords: preconcentration; dithizone; flame atomic absorption spectrometry; natural waters.

\section{INTRODUÇÃO}

Embora o desenvolvimento da instrumentação tenha possibilitado avanços em muitos aspectos da Química Analítica, em muitos casos a instrumentação disponível não apresenta sensibilidade analítica suficiente para a determinação de traços. Além disso, é comum não se analisar quimicamente amostras em sua forma original (aquosa ou sólida), pois elas podem conter espécies interferentes e serem incompatíveis com os equipamentos analíticos. Para contornar tais problemas são empregados procedimentos de preparo da amostra, os quais incluem várias etapas: coleta, armazenagem, solubilização, extração, pré-concentração, isolamento dos compostos de interesse e análise qualitativa e quantitativa. As etapas de extração e pré-concentração dos analitos são ainda o "calcanhar de Aquiles" de todo o procedimento analítico devido ao fato dessas etapas serem lentas, apresentarem a possibilidade de contaminação e a perda das espécies de interesse durante o manuseio da amostra. Assim, o desenvolvimento de procedimentos para a separação e pré-concentração de contaminantes de matrizes ambientais têm sido propostos, como por exemplo, extração líquido-líquido ${ }^{1-4}$, co-precipitação $0^{5}$, extração ponto nuvem $^{6}$, resinas trocadoras de íons ${ }^{7}$, eletrólises ${ }^{8}$ e extração em fase sólida $^{9-11}$. Entre estes procedimentos, a extração líquido-líquido é freqüentemente utilizada como técnica de separação. Apesar de sua eficiência na remoção de interferentes e na pré-concentração de analitos, o processo de extração líquido-líquido é lento e responsável pela geração de grandes volumes de resíduos de solventes orgânicos.

A micro extração é uma forma de extração líquido-líquido convencional na qual a extração é realizada com razão entre fases superior a $100^{12}$. Comparada com a extração convencional, a micro extração se caracteriza por piores recuperações do analito, mas a concentração na fase orgânica é aumentada. Adicionalmente, o volume de solvente orgânico usado é reduzido e somente uma etapa de manipulação é necessária, o que reduz problemas de contaminação e perdas de analitos.

*e-mail: carasek@qmc.ufsc.br
Muitos trabalhos têm sido descritos para a pré-concentração de poluentes orgânicos de matrizes aquosas utilizando micro extração líquido-líquido. Entretanto, apesar das vantagens desta técnica, poucos trabalhos têm sido publicados para a pré-concentração de metais via micro extração líquido-líquido. O processo de extração ocorre pelo contato de um elevado volume de amostra com uma alíquota do solvente orgânico contendo o agente complexante, na qual ocorre o enriquecimento do analito. Luque Perez et al..$^{13}$ desenvolveram um procedimento de micro extração líquido-líquido para o monitoramento contínuo de chumbo. Este método foi baseado na determinação espectrofotométrica de chumbo complexado com ditizona dissolvida em tetracloreto de carbono. Mais recentemente, Carasek ${ }^{14}$ demonstrou a viabilidade do uso do frasco de Murray modificado ${ }^{15}$ para pré-concentração de ouro em água de mar após complexação com dietilditiofosfato de amônio (DDTP). O complexo Au - DDTP foi extraído em $800 \mu \mathrm{L}$ de xileno e alíquotas de $100 \mu \mathrm{L}$ do extrato foram injetadas diretamente no FAAS. O tempo de extração foi de 2 min e não houve outras etapas de manipulação da amostra. O uso do frasco de Murray modificado permitiu a obtenção de limite de detecção para ouro da ordem de $n g \mathrm{~L}^{-1}$, desvio padrão relativo $(\mathrm{n}=10)$ inferior a $3,5 \%$ e faixa dinâmica linear entre $0-10 \mu \mathrm{g} \mathrm{L}^{-1}$.

Este trabalho propõe um procedimento analítico rápido e de baixo custo para a pré-concentração de traços de chumbo e cádmio em águas naturais, utilizando o frasco de Murray modificado ${ }^{15}$, após complexação com ditizona dissolvida em xileno e determinação utilizando espectrometria de absorção atômica com chama.

\section{PARTE EXPERIMENTAL}

\section{Instrumentação}

Um espectrômetro de absorção atômica com chama (Hitachi modelo Z-8230) equipado com corretor de fundo Zeeman foi utilizado. Medidas de absorbância foram feitas a 283,3 nm (Pb) e 228,8 nm (Cd) usando lâmpadas de cátodo oco da Hitachi (Mitorika, Ibaraki, Japão) operando com 7,5 e 6,5 mA, respectivamente. Os parâmetros utiliza- 
dos foram aqueles recomendados pelo fabricante. Todas as medidas de absorbância foram realizadas em área de pico após a injeção de 100 $\mu \mathrm{L}$ de extrato através de um pequeno funil de $\mathrm{PTFE}^{\circledR}$ conectado ao nebulizador ${ }^{14}$. Este dispositivo consiste de um tarugo de PTFE $^{\circledR}$ de 2,5 $\mathrm{cm}$ de largura e $1,5 \mathrm{~cm}$ de altura, no qual foi feito um furo de $0,8 \mathrm{~mm}$ de diâmetro interno e de $1,0 \mathrm{~mm}$ em uma de suas extremidades, apresentando formato de funil, em seu interior. A saída deste dispositivo foi conectada ao tubo utilizado para a aspiração da amostra para o nebulizador do espectrômetro de absorção atômica com chama. O micro extrator líquido-líquido, mostrado na Figura 1, foi utilizado nos procedimentos de pré-concentração de chumbo e cádmio.

Um pHmetro Orion modelo $720 \mathrm{~A}$ foi usado para ajustes de $\mathrm{pH}$ do meio.

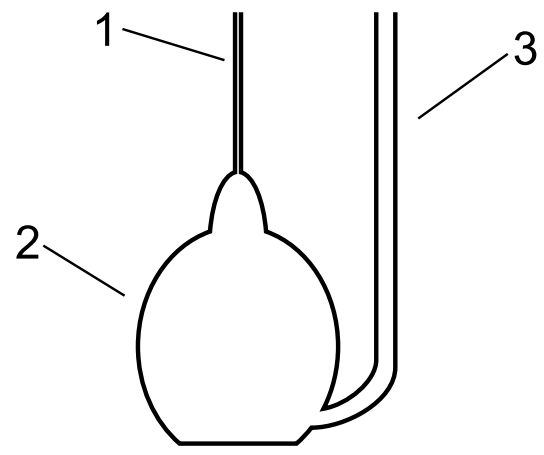

Figura 1. Frasco de microextração líquido-líquido: 1 = tubo capilar (diâmetro interno de $3 \mathrm{~mm}$ e altura de $10 \mathrm{~cm}$ ), 2 = frasco de $1 \mathrm{~L}$ modificado e 3 = braço lateral (diâmetro interno de $20 \mathrm{~mm}$ e altura de $30 \mathrm{~cm}$ )

\section{Reagentes}

Todos os reagentes utilizados foram de grau analítico e a água utilizada na preparação de todas as soluções de trabalho foi obtida do sistema de purificação Milli-Q (Millipore, Bedford, MA, EUA). As vidrarias utilizadas permaneceram imersas em solução de ácido nítrico $10 \% \mathrm{v} / \mathrm{v}$ durante a noite. Em seguida, foram enxaguadas com água desionizada e colocadas em um ambiente isento de poeira para secagem. As soluções de trabalho foram preparadas no mesmo dia da análise, através de apropriada diluição das soluções estoque de chumbo $\left(\mathrm{Pb}\left(\mathrm{NO}_{3}\right)_{2}\right.$ em solvente $\left.\mathrm{HNO}_{3}\right)$ e cádmio $\left(\mathrm{Cd}\left(\mathrm{NO}_{3}\right)_{2} \cdot 4 \mathrm{H}_{2} \mathrm{O}\right.$ em solvente $\mathrm{HNO}_{3}$ ) com concentração de $1000 \mu \mathrm{g} \mathrm{mL}-1$ cada. As soluções de chumbo e cádmio tiveram o pH ajustado com soluções tampão de acetato ou de amônio. Esses tampões foram preparados pela adição de solução de ácido acético (Vetec) na amostra até que o $\mathrm{pH}$ da mesma atingisse uma unidade abaixo daquele desejado e, em seguida, pela adição de solução de hidróxido de amônio (Grupo Química) na amostra até que o pH atingisse o valor desejado. Neste procedimento o volume total das soluções adicionadas foi inferior a $2 \mathrm{~mL}$. Procedimento idêntico foi realizado para a obtenção do tampão de amônio. As concentrações das soluções utilizadas para ajuste do $\mathrm{pH}$ estão descritas no item procedimento. Solução de ditizona (100,0 $\mathrm{mg} \mathrm{L}^{-1}$ ) foi preparada pela dissolução de $2,5 \mathrm{mg}$ de ditizona (ACS) em $25 \mathrm{~mL}$ de xileno (Grupo Química) e usado como agente complexante e solvente extrator.

\section{Procedimento}

Amostras de água $(500 \mathrm{~mL})$ devidamente tamponadas com volumes apropriados de solução de ácido acético $1,0 \mathrm{~mol} \mathrm{~L}^{-1}$ e solução de hidróxido de sódio $0,002 \mathrm{~mol} \mathrm{~L}^{-1}$ (ou solução de cloreto de amônio $1,0 \mathrm{~mol} \mathrm{~L}^{-1}$ e solução de ácido clorídrico $0,1 \mathrm{~mol} \mathrm{~L}^{-1}$ ) foram transferidas, através do braço lateral do micro extrator, para o frasco de Murray modificado com capacidade para $1000 \mathrm{~mL}$. De maneira similar, $2500 \mu \mathrm{L}$ de solução de ditizona $100,0 \mathrm{mg} \mathrm{L}^{-1}$ foram introduzidos no micro extrator. Então, a mistura foi agitada manualmente durante 4 min (tempo otimizado) para a complexação dos metais. No equilíbrio, uma porção do solvente orgânico se dissolveu na fase aquosa devido à sua miscibilidade. A camada de fase orgânica imiscível restante foi deslocada para o tubo capilar através da adição de água pelo braço lateral. Quando $2500 \mu \mathrm{L}$ de xileno foram utilizados para a extração, aproximadamente $1800 \mu \mathrm{L}$ puderam ser recuperados. Com o auxílio de uma micro pipeta foram retirados aproximadamente $500 \mu \mathrm{L}$ do solvente extrator, o qual foi um volume suficiente para a realização de 3 injeções de $100 \mu \mathrm{L}$ no espectrômetro de absorção atômica com chama. Os sinais obtidos para cada extrato foram utilizados sem nenhuma correção de volume. Soluções de trabalho de chumbo e cádmio em concentrações de, respectivamente, 10,0 - 80,0 $\mu \mathrm{g} \mathrm{L}^{-1}$ e 0,3 - 3,0 $\mu \mathrm{g} \mathrm{L}^{-1}$ foram extraídas, separada e simultaneamente, de maneira similar. Os resultados foram utilizados para construção de curvas analíticas. Brancos foram obtidos em paralelo para todas as determinações.

Os parâmetros experimentais do micro extrator líquido-líquido foram otimizados através do método univariado, ou seja, fixava-se os valores de todas as variáveis, exceto aquela em estudo. Uma vez otimizada uma variável, nos estudos seguintes, ela era mantida em seu valor otimizado. A otimização foi realizada considerando-se o máximo sinal e a melhor repetibilidade para extratos obtidos pela pré-concentração de soluções de chumbo e cádmio com concentrações de, respectivamente, $60,0 \mu \mathrm{g} \mathrm{L}^{-1}$ e $1,0 \mu \mathrm{g} \mathrm{L}^{-1}$. Todos os procedimentos para otimização foram realizados separadamente, e em triplicata, para soluções de chumbo e cádmio utilizando xileno como solvente extrator. Esse solvente foi utilizado devido à baixa solubilidade e menor densidade que a água, facilitando o uso do frasco de Murray modificado. Além disto, ditizona apresenta apropriada dissolução em xileno para a extração dos metais contidos nas amostras aquosas.

\section{Amostras}

Amostras de água (2000 mL) foram coletadas da Lagoa do Peri em Florianópolis - SC, Brasil. Estas amostras foram filtradas através de filtros de membrana de celulose $0,45 \mu \mathrm{m}$ da Millipore. Amostras de água de torneira e água mineral também foram coletadas e utilizadas tal qual. As amostras foram estocadas em frascos de polietileno e mantidas em baixa temperatura $\left( \pm 4{ }^{\circ} \mathrm{C}\right)$ previamente ao procedimento de micro extração, o qual foi realizado no dia seguinte.

\section{RESULTADOS E DISCUSSÃO}

\section{Otimização das condições operacionais}

O efeito do pH sobre a eficiência da micro extração foi estudado através de extrações de $500 \mathrm{~mL}$ de amostras de água desionizada contendo $30 \mu \mathrm{g}$ de chumbo e $0,5 \mu \mathrm{g}$ de cádmio com diferentes valores de $\mathrm{pH}$. De acordo com os resultados, mostrados na Figura 2a, os valores de $\mathrm{pH}$ ótimos para extrações de chumbo e cádmio são, respectivamente, 5,0 e 6,0. Os baixos sinais de absorbância para chumbo e para cádmio nos extratos orgânicos, os quais foram obtidos pelo procedimento de micro extração líquido-líquido em amostras aquosas com pH maior do que 8, podem ser atribuídos à dissolução da ditizona sob meio alcalino ${ }^{16}$. Por outro lado, a redução no sinal de absorbância do chumbo e do cádmio nos extratos orgânicos obtidos 
das extrações de amostras aquosas com $\mathrm{pH}$ menores que 4 sugere que íons $\mathrm{H}^{+}$poderiam protonar os nitrogênios com pares de elétrons disponíveis da ditizona impossibilitando a complexação dos metais chumbo e cádmio ${ }^{16}$. Outra possibilidade seria a ocorrência da ruptura do complexo metal - ditizona. Esta segunda possibilidade foi testada analisando-se os extratos orgânicos e os extratos aquosos ácidos obtidos após procedimentos de extração de retorno. Nestes procedimentos, $1800 \mu \mathrm{L}$ de extratos orgânicos obtidos com o uso do frasco de Murray modificado foram extraídos com $800 \mu \mathrm{L}$ de soluções de ácido nítrico $1 \mathrm{~mol} \mathrm{~L}^{-1}$. A análise dos extratos orgânicos obtidos após extrações de retorno, não apresentou sinais para chumbo e cádmio. Por outro lado, a análise dos extratos aquosos apresentou elevados sinais para chumbo e cádmio.

O tempo de agitação para extração de chumbo e cádmio também foi estudado. Os resultados indicaram um aumento do sinal para os extratos de chumbo e cádmio com o aumento do tempo de agitação de até 2 min, atingindo valores constantes de sinal após esse intervalo de agitação. Um tempo de agitação de 4 min foi selecionado.

Para a definição da concentração de ditizona necessária para a extração de chumbo e cádmio, o procedimento proposto foi aplicado variando-se a concentração da solução de ditizona entre 5,0 e 100,0 $\mathrm{mg} \mathrm{L}^{-1}$. Foi verificado que a eficiência da extração de chumbo e de cádmio aumentou com o aumento na concentração do agente complexante, atingindo um valor máximo e constante de absorbância acima de $80,0 \mathrm{mg} \mathrm{L}^{-1}$ (Figura 2b). Assim, a concentração de ditizona recomendada é de $100,0 \mathrm{mg} \mathrm{L}^{-1}$. Esta concentração é suficiente para a pré-concentração de chumbo e cádmio de águas naturais, pois as concentrações destes metais nestas amostras são geralmente baixas. Um excesso de ditizona foi utilizado porque outros metais complexam com este agente quelante. Este agente tem sido utilizado para a separação e pré-concentração de aproximadamente 20 outros metais ${ }^{17-19}$ em uma variedade de amostras para subseqüente determinação dos analitos por espectrometria de absorção atômica ${ }^{9,11,13}$.

$\mathrm{O}$ efeito da razão de volumes da fase aquosa e fase orgânica $\left(\mathrm{V}_{\mathrm{aq}} /\right.$ $\mathrm{V}_{\text {org }}$ ) foi investigado usando $500 \mathrm{~mL}$ de água desionizada contendo separadamente $30 \mu \mathrm{g}$ de chumbo e $0,5 \mu \mathrm{g}$ de cádmio, com valores otimizados de $\mathrm{pH}$, tempo de agitação e concentração de ditizona. Os resultados são mostrados na Figura 2c. No caso da extração de chumbo, a redução nos sinais de absorbância para volumes menores que $2000 \mu \mathrm{L}$ sugere que não há suficiente agente quelante para a complexação do metal. Para volumes de xileno relativamente elevados (maior que $2000 \mu \mathrm{L}$ ), houve uma redução linear no sinal devido à diluição do extrato. No caso da extração de cádmio, houve uma relação linear entre o sinal e os volumes de xileno utilizados neste estudo. Portanto, é possível aumentar o fator de pré-concentração reduzindo o volume de solvente extrator.

\section{Estudo de interferentes}

A influência de alguns íons que poderiam reagir com a ditizona foi investigada com o objetivo de identificar alguns potenciais interferentes. Neste estudo, foi utilizado um nível de tolerância na concentração dos íons interferentes definido como a máxima concentração que causaria uma mudança no sinal de, pelo menos, $10 \%$ comparado com o sinal de chumbo ou cádmio isoladamente. Foram utilizados parâmetros otimizados do micro extrator líquido-líquido e amostras de água contendo 20,0 e 1,0 $\mu \mathrm{g} \mathrm{L}^{-1}$ de chumbo e cádmio, respectivamente. Os íons investigados foram $\mathrm{Na}^{+}, \mathrm{Cl}^{-}$e $\mathrm{PO}_{4}^{3-}$, os quais podem estar presentes em altas concentrações em amostras ambientais. Os íons $\mathrm{Fe}^{3+} \mathrm{e}^{3+}$, os quais podem formar complexos com a ditizona, também foram investigados. Nenhuma interferência sobre o processo de micro extração foi observada quando adicionado $\mathrm{Na}^{+}$até a concentração de $65 \mathrm{~g} \mathrm{~L}^{-1}, \mathrm{Cl}^{-}$até $100 \mathrm{~g} \mathrm{~L}^{-1}$ e $\mathrm{PO}_{4}^{3-}$ até a concentração de
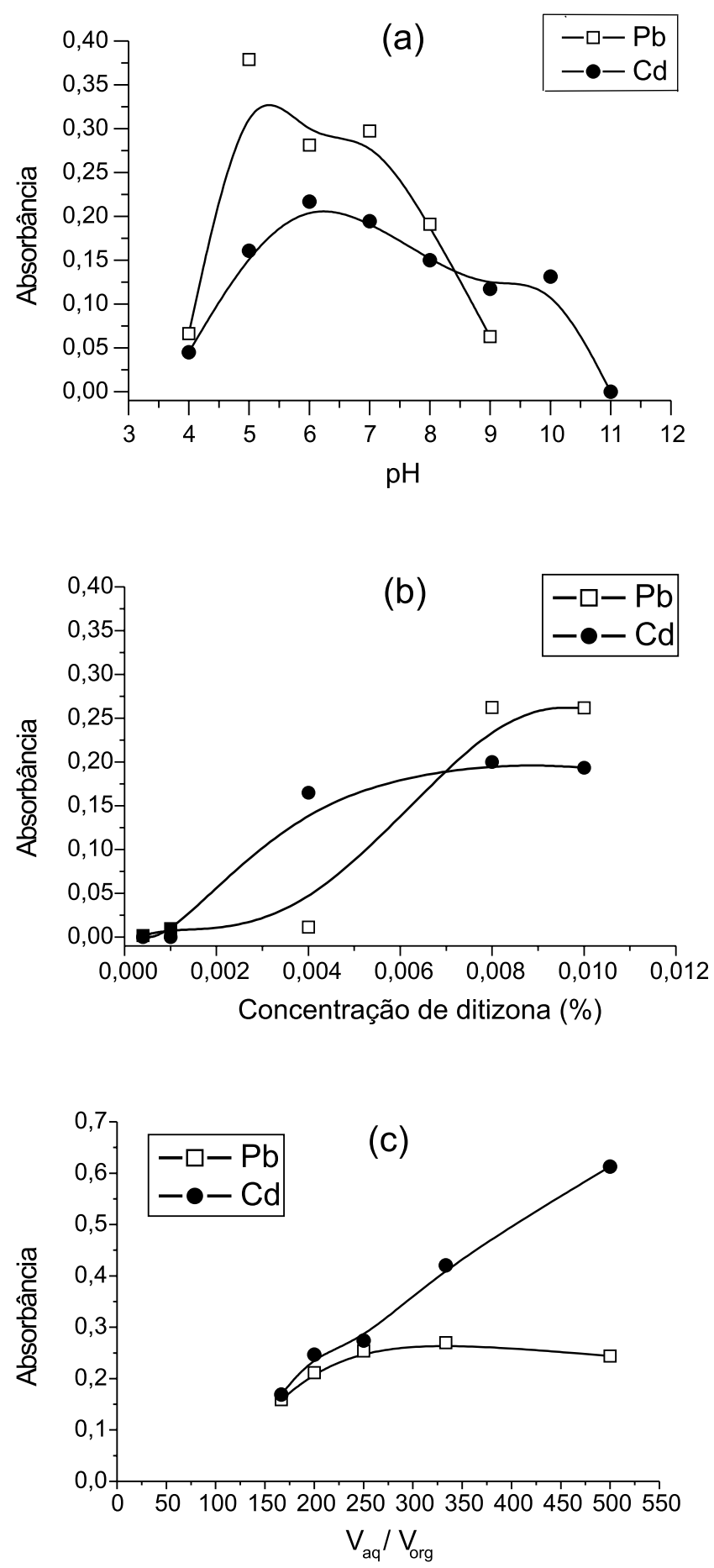

Figura 2. (a) Efeito do pH da amostra, (b) influência da concentração de ditizona e (c) efeito da relação entre volume de amostra e volume de solvente extrator sobre a absorbância do $\mathrm{Pb}$ e Cd após o procedimento de extração. Volumes de amostra de $500 \mathrm{~mL}$, concentrações de 60,0 $\mu \mathrm{g} \mathrm{L}^{-1}$ e 1,0 $\mu \mathrm{g} \mathrm{L}^{-1}$ para, respectivamente, chumbo e cádmio, tempo de agitação de 4 min e injeção de $100 \mu \mathrm{L}$ no FAAS

$5 \mathrm{mg} \mathrm{L}^{-1}$. Entretanto, houve interferência no processo de micro extração na presença de $\mathrm{Fe}^{3+}$ e $\mathrm{Al}^{3+}$ em níveis superiores a, respectivamente, $10,0 \mu \mathrm{g} \mathrm{L}^{-1}$ e $80,0 \mu \mathrm{g} \mathrm{L}^{-1}$. Este efeito pode ter ocorrido devido à concentração insuficiente do agente quelante para formar ditizonatos dos analitos de interesse e de ferro e alumínio. Outra possibilidade, seria devido ao fato do íon $\mathrm{Al}^{3+}$ ser um íon ácido fraco, que em solu- 
ção aquosa sofre solvatação. O íon $\left[\mathrm{Al}\left(\mathrm{H}_{2} \mathrm{O}\right)_{6}\right]^{3+}$ ao sofrer hidrólise, libera um $\mathrm{H}^{+}$que poderia protonar os nitrogênios com pares de elétrons disponíveis da ditizona, impossibilitando-a de complexar os metais chumbo e cádmio ${ }^{20}$. Entretanto, estas interferências podem ser atenuadas pela adição de citrato que complexa com íon $\mathrm{Fe}^{3+} \mathrm{e}$ $\mathrm{Al}^{3+}$. Para uma amostra contendo $200,0 \mu \mathrm{g} \mathrm{L}^{-1} \mathrm{Fe}^{3+}$, o qual é a concentração máxima total de Fe permitida em águas de abastecimento público ${ }^{21}$, a adição de $2,5 \mathrm{mg} \mathrm{L}^{-1}$ de citrato eliminou a interferência de $\mathrm{Fe}^{3+}$ no processo de micro extração proposto (Figura 3 ).

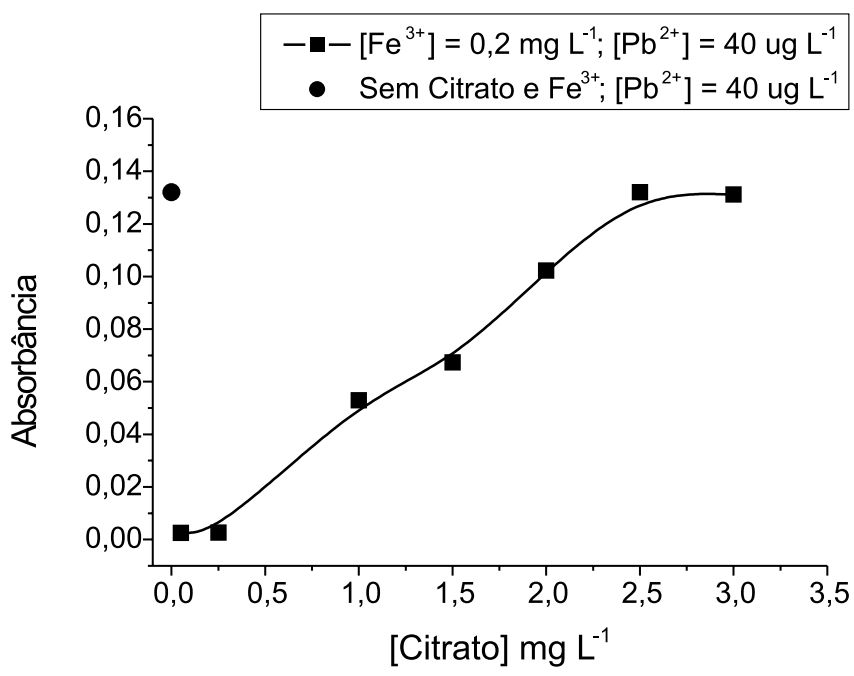

Figura 3. Efeito da adição de citrato na extração de $500 \mathrm{~mL}$ de amostra contendo $40 \mu \mathrm{g} L^{-1}$ de chumbo e 0,200 $\mathrm{mg} \mathrm{L}^{-1}$ de $\mathrm{Fe}^{3+}$. Tempo de agitação de 4 min e injeção de $100 \mu \mathrm{L}$ do extrato no FAAS

\section{Características analíticas do método}

Curvas analíticas foram construídas pela introdução de $100 \mathrm{~mL}$ dos extratos de xileno obtidos da pré-concentração de soluções-padrão de chumbo e cádmio, isoladamente e misturados, no espectrômetro de absorção atômica com chama. Linearidade, limite de detecção, tempo de extração e fator de pré-concentração para chumbo e cádmio estão listados na Tabela 1. Os valores apresentados foram obtidos utilizando-se as condições otimizadas do processo de micro extração. No caso de extrações simultâneas de chumbo e cádmio, foi utilizado $\mathrm{pH} 6$.

O limite de detecção foi estimado como 3 vezes o desvio padrão dos sinais de absorbância para injeções de $100 \mu \mathrm{L}$ de 7 extrações do branco usando o processo de micro extração. A precisão do método foi obtida através da estimativa do desvio padrão relativo (\%) de 7 soluções contendo $3,0 \mu \mathrm{g} \mathrm{L}^{-1}(\mathrm{~Pb})$ e $0,3 \mu \mathrm{g} \mathrm{L} \mathrm{L}^{-1}(\mathrm{Cd})$.

Para se avaliar a aplicabilidade do método proposto na determinação quantitativa de chumbo e cádmio em amostras de água, três diferentes amostras foram enriquecidas com diferentes concentrações de chumbo e cádmio. As amostras coletadas foram previamente submetidas ao procedimento de micro extração e foi determinado que o teor original de chumbo e cádmio estava abaixo do limite de detecção do método proposto. Além do estudo da recuperação analítica realizado com amostras de água enriquecidas com chumbo e cádmio, os resultados obtidos com o método proposto foram comparados com aqueles obtidos por uma metodologia alternativa de preparo de amostra (extração em fase sólida) ${ }^{22}$. Os resultados destes estudos estão apresentados na Tabela 2 e são indicativos de que os íons chumbo e cádmio podem ser quantitativamente recuperados das amostras testadas. Estes estudos foram alternativos ao uso de material de referência certificado, uma vez que o volume de amostra é elevado $(500 \mathrm{~mL})$.

O fator de enriquecimento foi determinado como a razão dos volumes de amostra e solvente extrator utilizados e, portanto, não foi levado em consideração o fator de recuperação do método.

\section{CONCLUSÕES}

O frasco de Murray modificado é útil para a pré-concentração e determinação de baixos níveis de chumbo e cádmio em amostras de água. O pequeno volume de solvente extrator, e por isso a denominação de micro extrator, possibilitou atingir elevados fatores de pré-concentração, os quais foram adequados para determinação por espectrometria de absorção atômica com chama. O tempo requerido para o procedimento completo de extração foi inferior a 5 min e ne-

Tabela 1. Determinação de $\mathrm{Pb}$ e $\mathrm{Cd}$ com a pré-concentração utilizando micro extração líquido-líquido

\begin{tabular}{|c|c|c|c|c|c|}
\hline Metal & Equação da reta ${ }^{a}$ & $\begin{array}{l}\text { Coeficiente de } \\
\text { correlação }\end{array}$ & $\begin{array}{l}\text { Faixa linear } \\
\qquad\left(\mu \mathrm{g} \mathrm{L}^{-1}\right)\end{array}$ & $\begin{array}{l}\text { Limite de detecção } \\
\qquad\left(\mu \mathrm{g} \mathrm{L}^{-1}\right)\end{array}$ & $\operatorname{DPR}(\%)^{\mathrm{b}}$ \\
\hline $\mathrm{Pb}$ & $Y=0,0054+0,0024 X$ & 0,9998 & $10-80$ & 1,56 & 5,8 \\
\hline $\mathrm{Cd}$ & $Y=-0,0092+0,14 X$ & 0,9982 & $0,3-3$ & 0,011 & 5,9 \\
\hline
\end{tabular}

a: absorbância; X: concentração do metal $\left(\mu \mathrm{g} \mathrm{L}^{-1}\right)$

${ }^{\mathrm{b}}$ Média de 7 determinações

Tabela 2. Determinação de $\mathrm{Pb}$ e Cd em amostras de águas naturais $(\mathrm{n}=3)$. Dados em $\mathrm{mg} \mathrm{L}^{-1}$

\begin{tabular}{|c|c|c|c|c|c|c|}
\hline \multirow{3}{*}{$\begin{array}{l}\text { Amostras } \\
\text { de água }\end{array}$} & \multicolumn{3}{|c|}{$\mathrm{Pb}$} & \multicolumn{3}{|c|}{$\mathrm{Cd}$} \\
\hline & \multirow[t]{2}{*}{ adicionado } & \multicolumn{2}{|c|}{ encontrado } & \multirow[t]{2}{*}{ adicionado } & \multicolumn{2}{|c|}{ encontrado } \\
\hline & & Micro extração & Extração alternativa & & Micro extração & Extração alternativa \\
\hline \multirow[t]{2}{*}{ Lagoa do Peri } & 40,0 & $39,0 \pm 2,7$ & $39,2 \pm 1,9$ & 2,0 & $1,9 \pm 0,1$ & $2,0 \pm 0,1$ \\
\hline & 100,0 & $102,0 \pm 7,1$ & $97,0 \pm 4,8$ & 4,0 & $3,8 \pm 0,3$ & $4,2 \pm 0,2$ \\
\hline \multirow[t]{2}{*}{ de torneira } & 40,0 & $39,4 \pm 1,9$ & $40,2 \pm 1,6$ & 2,0 & $2,2 \pm 0.1$ & $1,8 \pm 0,1$ \\
\hline & 100,0 & $101,0 \pm$ & $99,6 \pm 5,9$ & 4,0 & $3,8 \pm 0,3$ & $3,8 \pm 0,2$ \\
\hline \multirow[t]{2}{*}{ mineral } & 40,0 & $39,2 \pm 4,0$ & $40,6 \pm 2,0$ & 2,0 & $1,8 \pm 0,1$ & $2,0 \pm 0.1$ \\
\hline & 100,0 & $101,2 \pm 5,0$ & $98,8 \pm 4,9$ & 4,0 & $4,2 \pm 0,3$ & $3,8 \pm 0.2$ \\
\hline
\end{tabular}


nhuma etapa adicional de manipulação foi necessária, minimizando problemas de contaminação e perdas de analitos. A recuperação obtida, determinada através do método de adição e recuperação dos íons $\mathrm{Pb}^{2+}$ e $\mathrm{Cd}^{2+}$ e através da comparação com um método alternativo de preparo de amostra, permitiu a aplicabilidade do micro extrator líquido-líquido em amostras reais. Além disto, o custo da fabricação do frasco de Murray modificado é baixo e o procedimento de extração é simples, não necessitando de acessórios, como bombas peristálticas e válvulas comutadoras, como é o caso de procedimentos em fluxo contínuo. O elevado fator de enriquecimento obtido, quando utilizado o frasco de Murray modificado, permite o uso do espectrômetro de absorção atômica com chama, cujo custo de aquisição e manutenção é baixo, e portanto mais acessível, quando comparado a equipamentos como o espectrômetro de absorção atômica com forno de grafite. Desta forma, a opção pelo uso do frasco de Murray modificado é um compromisso entre as condições instrumentais do laboratório e a quantidade e tipo de amostra a ser analisada.

\section{AGRADECIMENTOS}

Os autores agradecem à Central de Análises do Departamento de Química da UFSC pelo suporte instrumental e à Coordenação de Aperfeiçoamento de Pessoal de Nível Superior/Fundação de Ciência e Tecnologia/Mestrado Interinstitucional UFSC-FURB pelo suporte financeiro.

\section{REFERÊNCIAS}

1. Dapaah, A.R.K.; Takano, N.; Ayame A.; Anal. Chim. Acta 1999, 386, 281.

2. Bortoli, A.; Gerotto, M.; Mariconti, F.; Palonta, M.; Troncon, A.; Microchem. J. 1996, 54, 402.
3. Dabeka, R.W.; Conacher, H.B.S.; Salminen, J.; Nixon, G.R.; Riedel, G.; Crocker, R.; Dube, G.; J. AOAC Int. 1992, 75, 449.

4. Saran, R.; Basu Baul, T.S.; Srinivas, P.; Khathing, D.T.; Anal. Lett. 1992, $25,1545$.

5. Martinez-Jimenez, P.; Gallego, M.; Valcárcel, M.; Analyst 1987, 112, 1233.

6. Silva, M.A.M.; Frescura, V.L.A.; Aguilera, F.J.N.; Curtius, A.J.; J. Anal. At. Spectrom. 1998, 13, 1369.

7. Packer, A.P.; Giné, M.F.; Miranda, C.E.S.; Reis, B.F.; J. Anal. At. Spectrom. 1997, 12, 563.

8. Batley, G.E.; Matousek, J.P.; Anal. Chem. 1977, 49, 2031.

9. Mahmoud, M.E.; Osman, M.M.; Amer, M.E.; Anal. Chim. Acta 2000, 415, 33.

10. Ferreira, S.L.C.; dos Santos, H.C.; Ferreira, J.R.; de Araujo, N.M.L.; Costa, A.C.S.; de Jesus, D.S.; J. Braz. Chem. Soc. 1998, 9, 525.

11. Manzoori, J.L.; Sonouraddin, M.H.; Shabani, A.M.H.; J. Anal. At. Spectrom. 1998, 13, 305.

12. Majors, R.E.; $L C$-GC 1996, 14, 936

13. Luque Perez, E.; Rios, A.; Valcárcel, M.; Quim. Anal. 1997, 16, 107.

14. Carasek, E.; Talanta 2000, 51, 173.

15. da Rocha, E. C.; Tese de Doutorado, Universidade Estadual de Campinas, Brasil, 1997.

16. Bassett, J.; Denney, R.C.; Jeffery, G.H.; Mendham, J.; Vogel: Análise Inorgânica Quantitativa, Ed. Guanabara Dois S.A.: Rio de Janeiro, 1981, p. 115.

17. Crosby, N.T.; Analyst 1977, 102, 233.

18. Dean, J.A.; Analytical Chemistry Handbook, Mc Graw-Hill: New York, 1995.

19. Yan, L.; Ingle Jr., J.D.; Anal. Chem. 1989, 61, 520.

20. Cotton, A.F.; Wilkinson, G.; Advanced Inorganic Chemistry: the GroupIII (13) Elements, Wiley-Interscience Publication: New York, 1988, p. 216.

21. Dajra - Adad, J.M.; Controle Químico de Qualidade, Ed. Guanabara Dois S.A.: Rio de Janeiro, 1982, p. 74 e 75.

22. Budziak, D.; Silva, E.L.; Campos, S.D.; Carasek, E.; Resumos da $24^{a}$ Reunião Anual da Sociedade Brasileira de Química, Poços de Caldas, Brasil, 2001. 